\title{
(2) OPEN ACCESS \\ Muscle-strengthening activities are associated with lower risk and mortality in major non-communicable diseases: a systematic review and meta-analysis of cohort studies
}

\author{
Haruki Momma 다, ${ }^{1}$ Ryoko Kawakami 다, ${ }^{2}$ Takanori Honda (다, \\ Susumu S Sawada (i) ${ }^{2}$
}

\begin{abstract}
- Additional supplemental material is published online only. To view, please visit the journal online (http://dx.doi. org/10.1136/bjsports-2021 105061).
\end{abstract}

${ }^{1}$ Department of Medicine and Science in Sports and Exercise, Tohoku University Graduate School of Medicine, Sendai, Miyagi, Japan

${ }^{2}$ Faculty of Sport Sciences, Waseda University, Tokorozawa, Saitama, Japan

${ }^{3}$ Department of Epidemiology and Public Health, Kyushu University Graduate School of Medical Sciences, Fukuoka, Japan

\section{Correspondence to}

Dr Haruki Momma, Department of Medicine and Science in Sports and Exercise, Tohoku University Graduate School of Medicine, Sendai, Miyagi, Japan; h-momma@med.tohoku.ac.jp

HM and RK contributed equally.

Accepted 2 January 2022

\section{Check for updates}

(C) Author(s) (or their employer(s)) 2022. Re-use permitted under CC BY. Published by BMJ.

To cite: Momma $\mathrm{H}_{\text {, }}$ Kawakami R, Honda T, et al. Br J Sports Med Epub ahead of print: [please include Day Month Year]. doi:10.1136/ bjsports-2021-105061

\begin{abstract}
Objective To quantify the associations between muscle-strengthening activities and the risk of noncommunicable diseases and mortality in adults independent of aerobic activities.

Design Systematic review and meta-analysis of prospective cohort studies.
\end{abstract}

Data sources MEDLINE and Embase were searched from inception to June 2021 and the reference lists of all related articles were reviewed.

Eligibility criteria for selecting studies Prospective cohort studies that examined the association between muscle-strengthening activities and health outcomes in adults aged $\geq 18$ years without severe health conditions. Results Sixteen studies met the eligibility criteria. Muscle-strengthening activities were associated with a 10-17\% lower risk of all-cause mortality, cardiovascular disease (CVD), total cancer, diabetes and lung cancer. No association was found between muscle-strengthening activities and the risk of some site-specific cancers (colon, kidney, bladder and pancreatic cancers). Jshaped associations with the maximum risk reduction (approximately 10-20\%) at approximately 30-60 min/ week of muscle-strengthening activities were found for all-cause mortality, CVD and total cancer, whereas an Lshaped association showing a large risk reduction at up to $60 \mathrm{~min} /$ week of muscle-strengthening activities was observed for diabetes. Combined muscle-strengthening and aerobic activities (versus none) were associated with a lower risk of all-cause, CVD and total cancer mortality. Conclusion Muscle-strengthening activities were inversely associated with the risk of all-cause mortality and major non-communicable diseases including CVD, total cancer, diabetes and lung cancer; however, the influence of a higher volume of muscle-strengthening activities on all-cause mortality, CVD and total cancer is unclear when considering the observed J-shaped associations.

Systematic review registration PROSPERO CRD42020219808.

\section{INTRODUCTION}

Physical inactivity is a global public health problem. Several national and international physical activity guidelines recommend regular musclestrengthening activities for adults. ${ }^{1-5}$ For example, the recent WHO guidelines recommend that adults should perform muscle-strengthening activities $\geq 2$ days/week. ${ }^{4}$ Regular engagement in musclestrengthening activities (eg, resistance training) increases or preserves skeletal muscle strength, ${ }^{3}$ which has been shown to be inversely associated with mortality ${ }^{67}$ and the risk of non-communicable diseases (NCDs) such as cardiovascular disease (CVD) and cancer. ${ }^{7}$ Therefore, promoting musclestrengthening activities may help in reducing the risk of premature death and NCDs.

Compared with aerobic activities, musclestrengthening activities have been less frequently investigated in terms of their influence on the prevention of premature death and NCDs. Saeidifard et al conducted the first systematic review and meta-analysis of 11 published studies that focused on mortality. ${ }^{8}$ Although no clear association was observed between resistance training and mortality from CVD and cancer, resistance training was found to be inversely associated with all-cause mortality. ${ }^{8}$ Moreover, a recent meta-analysis that focused on cancer incidence and mortality showed that muscle-strengthening activities were associated with a lower incidence of kidney cancer. ${ }^{9}$ Although these findings suggested a favourable influence of muscle-strengthening activities on the risk of NCDs and mortality, the dose-response association was not quantified. In some countries such as Japan, ${ }^{10}$ a revision of the national physical activity guidelines is under way, and there is a debate regarding whether muscle-strengthening activities should be included in the guidelines. Existing physical activity guidelines primarily focus on the musculoskeletal health benefits of muscle-strengthening activities. ${ }^{11-13}$ A systematic evaluation of the associations of muscle-strengthening activities with mortality and NCDs will aid in determining whether musclestrengthening activities need to be included in the guidelines. In addition, investigating the doseresponse association is also necessary to determine the amount of muscle-strengthening activities that should be recommended for public health purposes. A recent narrative review suggested the existence of dose-response associations between musclestrengthening activities and mortality and major NCDs. ${ }^{14}$ With the increasing number of relevant cohort studies, it is now possible to systematically update and expand on previous reviews that did not directly provide the optimal dose of musclestrengthening activities. 
We therefore conducted a systematic review and meta-analysis of prospective cohort studies on muscle-strengthening activities and the risk of mortality and NCDs among adults aged $\geq 18$ years. In addition to examining the health benefits of engaging in muscle-strengthening activities compared with the absence of muscle-strengthening activities independent of aerobic activities, we quantified the dose-response association between musclestrengthening activities and health outcomes. We also focused on the additional benefits of combined muscle-strengthening and aerobic activities for health outcomes.

\section{METHODS}

This systematic review was performed following the MOOSE ${ }^{15}$ and PRISMA $2020^{16}$ guidelines and was registered a priori in the PROSPERO database (CRD42020219808).

\section{Data sources and searches}

A systematic literature search was conducted in MEDLINE and Embase from the inception of the databases to 25 October 2020. The search syntax was designed by professional research agencies (International Medical Information Centre, Tokyo, Japan and Inforesta Co Ltd, Tokyo, Japan) with input from two authors (HM and RK) (see online supplemental table 1). We focused on the literature on the association between musclestrengthening activities and health outcomes among adults aged $\geq 18$ years without diagnosed severe health conditions (eg, cancer or disability) at baseline. Studies were considered eligible if they (1) had a prospective observational design; (2) had a minimum follow-up period of 2 years; (3) examined the influence of muscle-strengthening activities on the outcomes independent of and in combination with aerobic activities; and (4) were published in English. We included studies that used any health outcomes except for those that used a surrogate marker as an outcome.

\section{Study selection}

To select articles for full-text reading, two authors (HM and RK) independently screened the titles and abstracts using EndNote X9.2 (Clarivate Analytics, Pennsylvania, USA) and Microsoft Excel (Microsoft Corporation, Redmond, Washington, USA) after the exclusion of duplicates. Articles with ambiguous eligibility were included in the full-text reading step. The two authors also independently performed full-text reading of each article and a hand-search of the reference lists in the selected articles. No additional studies were found. Disagreements were resolved through discussion. An update of the primary search was conducted in June 2021.

\section{Data extraction}

Three authors (HM, RK, and TH) independently extracted the following information from each eligible study after dividing the selected papers among them: first author, publication year, study location, cohort name, sex, age of participants, number of participants and person-years, years of follow-up, number of deaths, cause of death, number of incident outcomes, subtype of incident outcome, assessment details for outcomes, assessment details for muscle-strengthening activities, covariates included in the analyses, and effect estimates and 95\% confidence intervals (CIs) of mortality or incidence of NCDs. If relevant information about the assessment of outcomes and exposures was missing from the eligible studies, we obtained the information from other studies of the same cohort. The most adjusted effect estimates in the main and sensitivity analyses were extracted. For each study, one of the three authors extracted the data and the remaining two authors cross-checked the data. Disagreements were resolved through deliberation to achieve consensus. Because most of the studies eligible for our meta-analyses reported hazard ratios, if other effect estimates such as ORs were reported, we asked the corresponding authors to provide the hazard ratios. ${ }^{1718}$ Moreover, if information about the effect estimate was not reported, we asked the corresponding authors to provide the hazard ratios using a template. ${ }^{19-21}$ Three authors provided additional data. ${ }^{171920}$ When multiple articles involving the same cohort for the same outcome were identified, only data from the most recently published article were used. In all such cases, the most recently published articles had the largest number of cases in our systematic review. When the publication year was the same, the article with the largest number of participants and cases was included.

\section{Quality assessment}

The quality of the studies was assessed using a modification of the Newcastle-Ottawa Scale (NOS) for Quality Assessment of Prospective Cohort Studies (see online supplemental table 2). ${ }^{22}$ We excluded the 'representativeness of the exposed cohort' item of the original NOS because our quality assessment was planned to evaluate internal validity, not external validity. Therefore, 8 stars in total were achievable, and a higher score indicated higher study quality. HM and RK independently assessed the studies and resolved any inconsistencies through discussion.

\section{Data synthesis and analysis}

A meta-analysis was conducted if at least two studies reported the effect estimate for the same outcome. Reported hazard ratios were considered equivalent to relative risks (RRs). When only ORs were available, ${ }^{18}$ they were considered equivalent to RRs because the overall cumulative incidence of the outcome was relatively low (16.5\%). Although we tried to convert ORs to RRs, we could not obtain an assumed control risk from the study because the number of cases was not provided. We assessed the influence of the inclusion of this study by performing a leave-one-out analysis. For the meta-analysis of the influence of muscle-strengthening activities, the effect estimates for any muscle-strengthening activities compared with no musclestrengthening activities were combined using the random-effects model of DerSimonian and Laird. ${ }^{23}$ When the included studies had two or more exposed groups, the effect estimates among the exposed groups were synthesised to obtain a pooled effect estimate using a fixed-effects model with the inverse variance method. ${ }^{24} 25$

We also conducted a dose-response meta-analysis to investigate the influence of muscle-strengthening activities on health outcomes using the method described by Greenland and Longnecker ${ }^{26}$ and Orsini et al. ${ }^{27}$ This method allows estimating studyspecific linear trends (slopes) considering the covariance for each exposure category within each study because they are calculated relative to a common reference group. ${ }^{2627}$ The method requires data including distribution of cases, person-years and adjusted RR with 95\% CI across three or more quantitative categories. If only the total number of cases or person-years was reported, the distribution of cases or person-years was estimated using the total number of cases and person-years and the RR according to the previous study. ${ }^{28}$ If the total number of person-years was not reported, we approximated it by multiplying the total number of participants by the median or mean of the follow-up period. The median or mean of the time of muscle-strengthening activities 
within the exposure categories was assigned to the corresponding RR. If these were not reported, the midpoint between the lower and upper limits was calculated. For open-ended categories, we assumed that they had the same widths as the closest category. We used 'none' as the reference group, and there was no study in which the reference category was not the lowest category. The study-specific slopes were pooled using the DerSimonian and Laird random-effects model. ${ }^{23}$ A potential non-linear association was also examined using a restricted cubic spline model with three knots at fixed percentiles (10\%, 50\% and 90\%) of time of the exposure. ${ }^{29}$ Non-linearity was assessed by testing the null hypothesis that the coefficient of the second spline was equal to zero using a Wald test. ${ }^{29}$

The joint benefit of muscle-strengthening activities and aerobic activities was also examined using the studies that reported the effect estimates of both muscle-strengthening and aerobic activities. The categories of muscle-strengthening (eg, none vs any or $\geq 2$ vs $<2$ times/week) and aerobic activity (eg, $\geq 150$ vs $<150 \mathrm{~min} /$ week or low vs high) were defined on the basis of the included studies.

Statistical heterogeneity between studies was examined using Cochrane's Q test and $\mathrm{I}^{2}$ statistic. $\mathrm{I}^{2}$ statistic with values of $25 \%, 50 \%$ and $75 \%$ corresponded to low, moderate and high level of heterogeneity, respectively. ${ }^{30}$ To examine the effect of individual studies on the pooled point estimate and 95\% CI of each outcome, we performed a sensitivity analysis by serially excluding each study and evaluated the corresponding changes in the effect estimate (leave-one-out analysis).

Subgroup analyses were performed according to sex (men only, women only, or men and women), age (>65 or $\leq 65$ years), exposure assessment (post hoc, questionnaire or interview) and NOS quality score (post hoc, $<7$ or $\geq 7$ ). However, subgroup analyses according to age and sex with cancer as the outcome were not performed owing to insufficient data.

Publication bias was assessed by visually inspecting the funnel plots of estimates against the SE of each study and by using Egger's test of funnel plot asymmetry ${ }^{31}$ if the number of included studies was $\geq 10$. $^{32}$

All analyses were performed using Stata 17 (StataCorp, College Station, Texas, USA). Statistical significance was set at $\mathrm{p}<0.05$.

\section{Grading the evidence}

The Grading of Recommendations Assessment, Development and Evaluation (GRADE) approach was used to assess the overall certainty of evidence for outcomes. ${ }^{33-38}$ One reviewer (HM) assessed the certainty of the evidence while two reviewers (RK and $\mathrm{TH}$ ) examined and revised the certainty of assessments as necessary. A GRADE evidence profile was developed (see online supplemental table 3). ${ }^{39}$

\section{RESULTS}

\section{Literature search}

A total of 1252 records were identified through systematic searches in MEDLINE and Embase after the removal of duplicates. Of these, 47 records were retrieved for full-text review and 29 studies were eligible based on the inclusion criteria. ${ }^{17-21} 40-63$ Among them, although a total of 28 outcomes were reported, only nine outcomes (all-cause mortality, CVD, total cancer, diabetes and site-specific cancers (colon, kidney, bladder, lung and pancreatic cancers)) were examined in two or more studies. Therefore, 17 outcomes were excluded from our meta-analyses (see online supplemental table 4), resulting in the exclusion of
Identification of studies via databases and registers

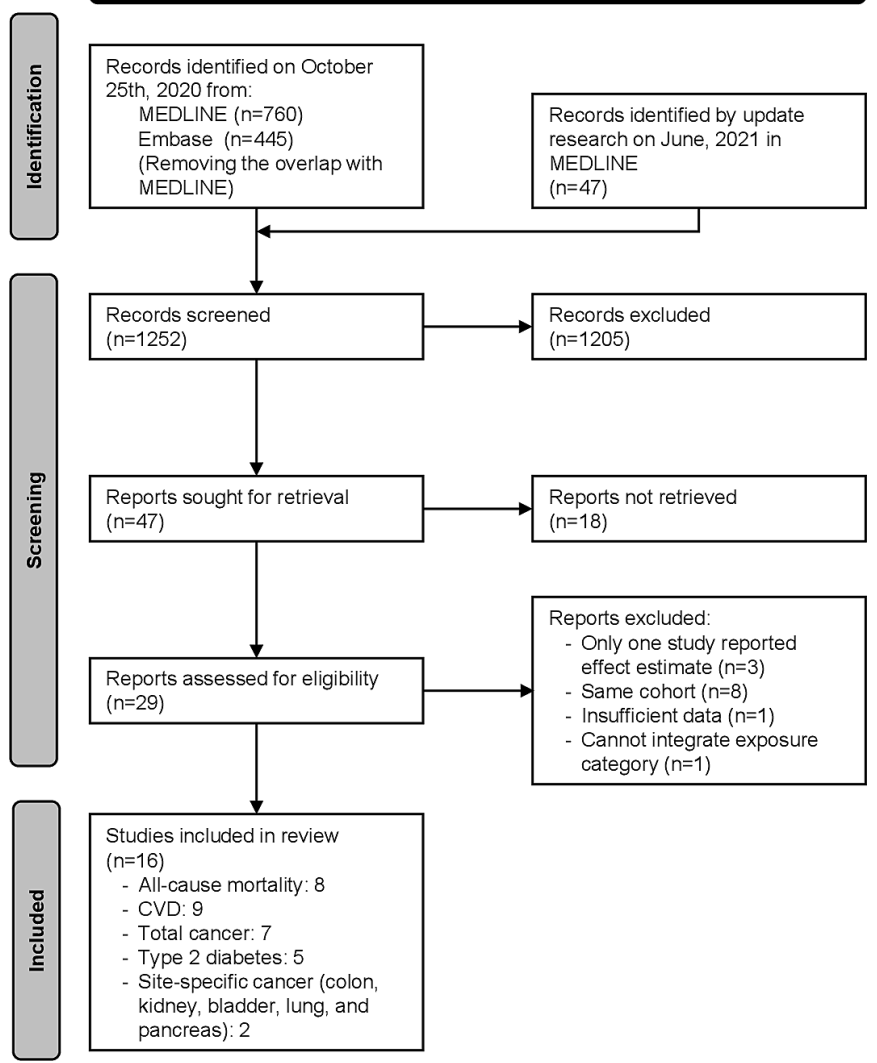

Figure 1 Flowchart of the selection of studies included in the metaanalysis.

three studies. ${ }^{60-62}$ Moreover, prostate cancer and lymphoma were also excluded because of discrepancies in the definition of outcomes across the studies. ${ }^{45} 50$ Of the remaining 26 studies we excluded eight because of multiple publications from the same cohort (see online supplemental table 5). ${ }^{52-59}$ One study was further excluded because of insufficient information about the effect estimate ${ }^{21}$ and another study was excluded because the exposure could not be integrated. ${ }^{63}$ Finally, 16 studies were included in the meta-analysis (figure 1). ${ }^{17-20} 40-51$

\section{Study characteristics}

The detailed characteristics of the studies included in the metaanalysis are presented in online supplemental table 6. The publication years ranged from 2012 to 2020. Most studies were conducted in the USA. ${ }^{18} 194042-51$ Other studies were from England and Scotland, ${ }^{20}$ Australia ${ }^{17}$ and Japan. ${ }^{41}$ The number of participants varied considerably (from 3809 to 479856 ). The maximum follow-up duration was 25.2 years (median). ${ }^{46}$ The age of participants ranged from 18 to 97.8 years. Twelve studies included both men and women, ${ }^{17} 18204144-4951$ two studies included men only ${ }^{1950}$ and three studies included women only. $^{40} 4243$ Adjustment for confounders varied widely across studies, with most studies adjusting for age, body mass index, alcohol intake and smoking status, whereas several studies adjusted for sex, race/ethnicity, dietary habits, disease history and sociodemographic status. All studies considered aerobic or other types of physical activity. Thirteen studies used selfreporting methods to measure muscle-strengthening activities $^{17-2040-464850}$ and three studies used interview methods. ${ }^{4749} 51$ All studies focused on muscle-strengthening exercises such as 
resistance/strength/weight training and callisthenics, but not on muscle-strengthening activities such as carrying heavy loads and heavy gardening.

\section{Risk of bias and certainty of evidence}

In the risk of bias assessment using the NOS (online supplemental table 2), the included studies were assigned 4-7 stars. For allcause mortality, four studies were assigned 7 stars, three studies were assigned 6 stars and one study was assigned 5 stars. For CVD, four studies were assigned 7 or 6 stars whereas one study was assigned 5 stars. For total cancer, four and three studies were assigned 7 and 6 stars, respectively, whereas one study was assigned 5 stars. For diabetes, four studies were assigned 6 stars and one study was assigned 4 stars.

The overall certainty of the evidence for each outcome and its details are shown in table 1 and online supplemental table 3. The grading of the certainty of the evidence was generally very low. The main reason for downgrading the evidence was indirectness because most of the studies included in this review were conducted in the USA.

\section{All-cause mortality}

Seven studies with 42133 cases of all-cause mortality among 263058 participants were included in the two-group analysis. Muscle-strengthening activities were associated with a $15 \%$ lower risk of all-cause mortality (RR 0.85 ; 95\% CI 0.79 to $0.93 ; \mathrm{p}<0.001$ ) (figure 2). Although the heterogeneity was high $\left(\mathrm{I}^{2}=83.0 \% ; \mathrm{p}<0.001\right)$, the association was in the same direction, with an RR of $<1.00$ in all studies. A similar result was obtained when Sheehan's study, ${ }^{18}$ which provided ORs, was excluded (RR $0.84 ; 95 \%$ CI 0.76 to 0.92 ; p < 0.001) (see online supplemental figure 1). Moreover, the exclusion of any other individual study did not substantially change this result, and the high heterogeneity was not explained by sex, quality score or exposure assessment (see online supplemental figures 1-4).

Six studies were eligible for the dose-response analysis of muscle-strengthening activities per $10 \mathrm{~min} /$ week increase, with a total of 236331 participants and 37178 cases. Although there was no clear linear association (figure 3), a non-linear association was observed (figure 4). The lowest RR (RR 0.83; 95\% CI 0.79 to 0.86 ) was observed at $40 \mathrm{~min} /$ week of muscle-strengthening activities, and the RR estimate for up to approximately $140 \mathrm{~min} /$ week was $<1.00$.

Three studies examined the joint benefit of musclestrengthening and aerobic activities for all-cause mortality, with a total of 581194 participants and 68637 cases. Combined muscle-strengthening and aerobic activities (vs none) were associated with a $40 \%$ lower risk of all-cause mortality (RR 0.60; 95\% CI 0.54 to $0.67 ; \mathrm{I}^{2}=59.3 \%$ ) (figure 5 ).

The overall quality of the evidence on all-cause mortality was rated as 'very low'.

\section{CVD}

Seven studies with 16056 cases of CVD among 257888 participants were included in the two-group analysis. Three studies focused on CVD mortality or CVD morbidity, ${ }^{43} 4446$ whereas other studies focused on CVD mortality. ${ }^{19} 2042484951$ Musclestrengthening activities were associated with a $17 \%$ lower risk of CVD (RR $0.83 ; 95 \%$ CI 0.73 to $0.93 ; p=0.002$ ), with a high level of heterogeneity $\left(\mathrm{I}^{2}=72.9 \% ; \mathrm{p}=0.001\right)$ (figure 2 ). Although the high heterogeneity was not completely explained by the quality score and exposure assessment, the heterogeneity disappeared $\left(\mathrm{I}^{2}=0.0 \%\right)$ when the study by Liu et $a l^{44}$ was excluded (online

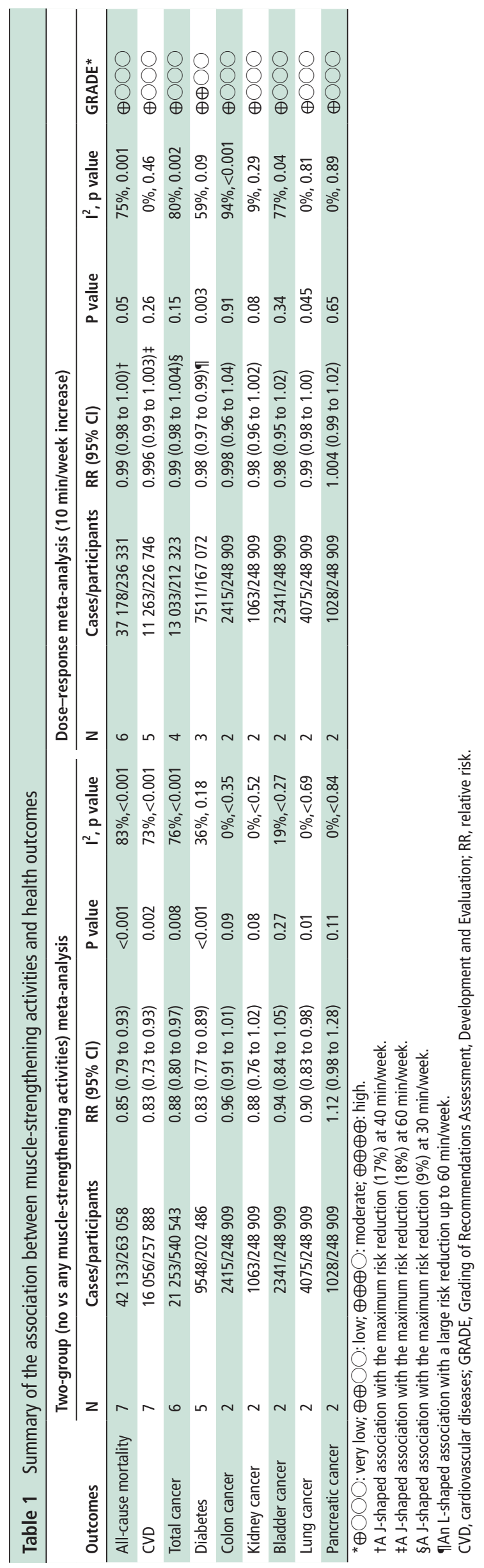


All-cause mortality
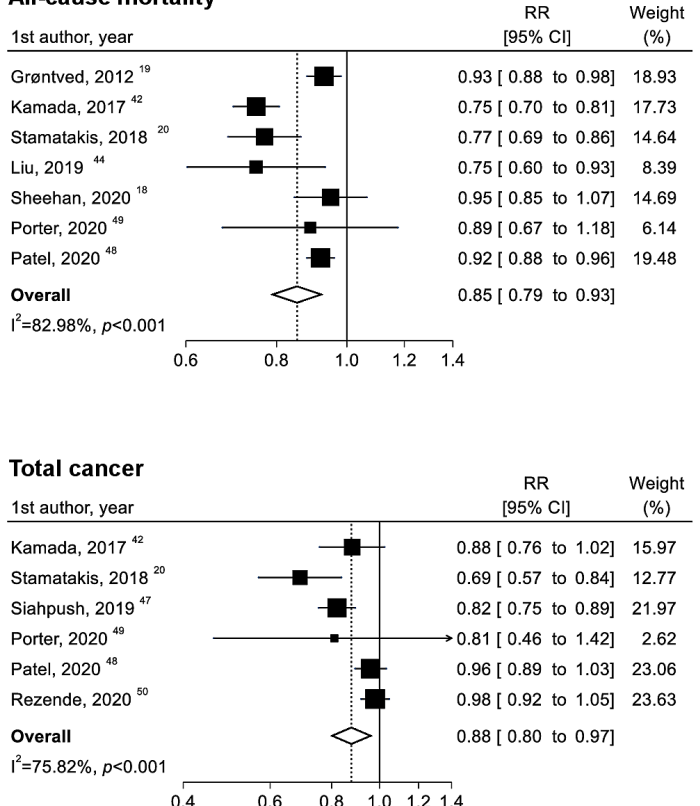

CVD

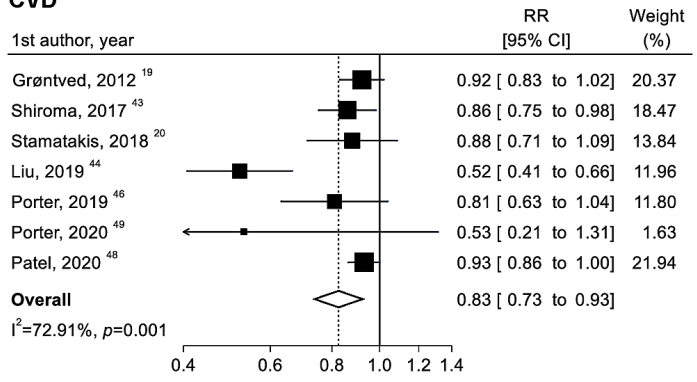

Diabetes

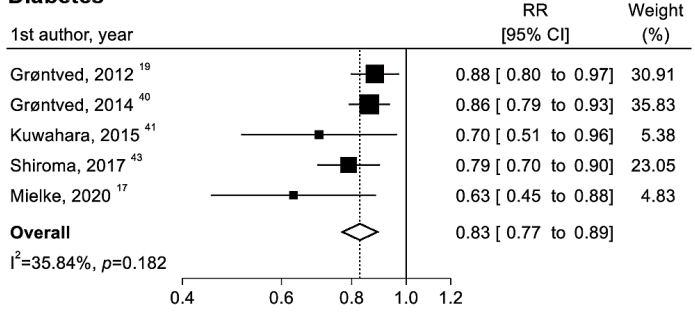

Figure 2 Two-group meta-analysis of the associations between no versus any muscle-strengthening activities and all-cause mortality, cardiovascular disease (CVD), total cancer and diabetes. RR, relative risk.

supplemental figure 1). Moreover, a similar result was obtained when the analysis was limited to CVD mortality (online supplemental figure 5).

Five studies were eligible for the dose-response analysis of muscle-strengthening activities per $10 \mathrm{~min} /$ week increase, with a total of 226746 participants and 11263 cases. Although there was no clear linear association (figure 3), a non-linear association was observed (figure 4). The lowest RR (RR 0.82; 95\% CI 0.76 to 0.90 ) was observed at $60 \mathrm{~min} /$ week of muscle-strengthening activities, and the RR estimate for up to approximately $130 \mathrm{~min} /$ week was $<1.00$.

Three studies examined the joint benefit of musclestrengthening and aerobic activities for CVD mortality, with a total of 582672 participants and 15643 cases. Combined
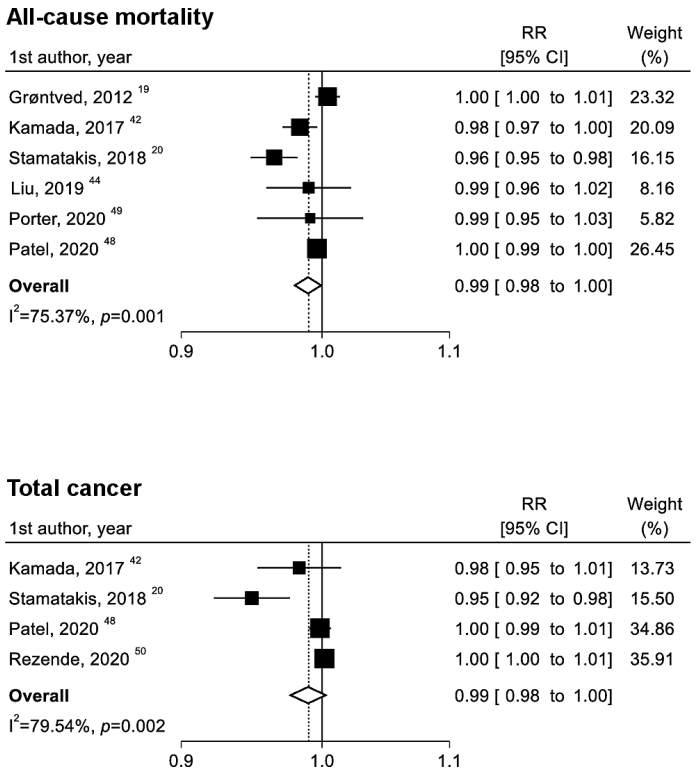

muscle-strengthening and aerobic activities were associated with a $46 \%$ lower risk of CVD (RR 0.54 ; $95 \%$ CI 0.41 to 0.70 ; $\mathrm{I}^{2}=62.6 \%$ ) (figure 5).

The overall quality of the evidence on CVD was rated as 'very low'.

\section{Total cancer}

Six studies with 21253 cases of total cancer among 540543 participants were included in the two-group analysis. One study focused on total cancer incidence, ${ }^{50}$ whereas the other studies focused on total cancer mortality. ${ }^{20} 42$ 47-49 Muscle-strengthening activities were associated with a $12 \%$ lower risk of total cancer (RR 0.88 ; 95\% CI 0.80 to 0.97 ; $=0.008$ ), with a high level of

CVD

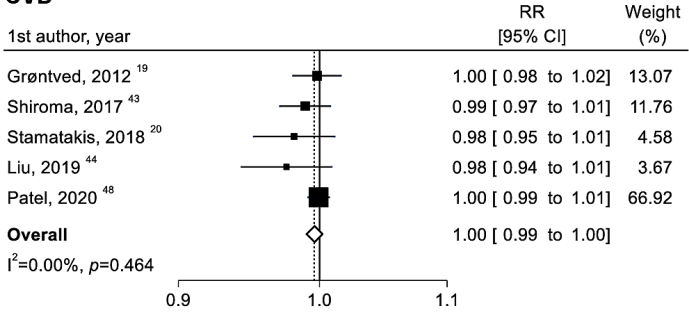

\begin{tabular}{l}
$\begin{array}{l}\text { Diabetes } \\
\text { 1st author, year }\end{array}$ \\
\hline $\begin{array}{l}\text { Grøntved, } 2012^{19} \\
\text { Grøntved, } 2014^{40}\end{array}$ \\
Shiroma, 2017 \\
Overall \\
$I^{2}=58.69 \%, p=0.089$
\end{tabular}

Figure 3 Linear dose-response meta-analysis of the associations between muscle-strengthening activities (per 10 min/week increase) and all-cause mortality, cardiovascular disease (CVD), total cancer and diabetes. RR, relative risk. 

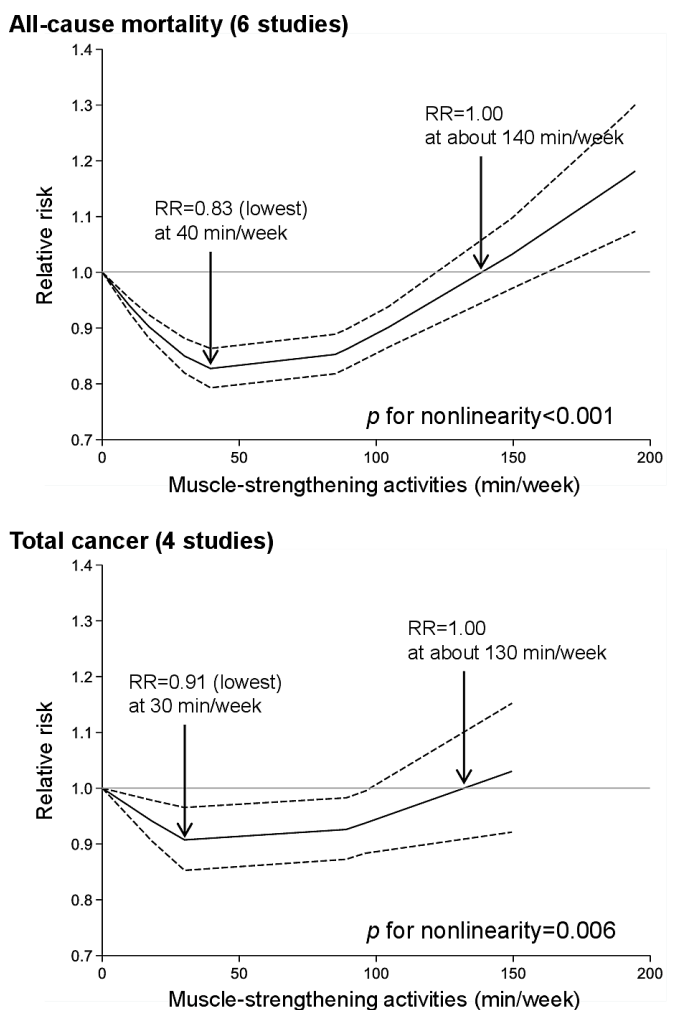

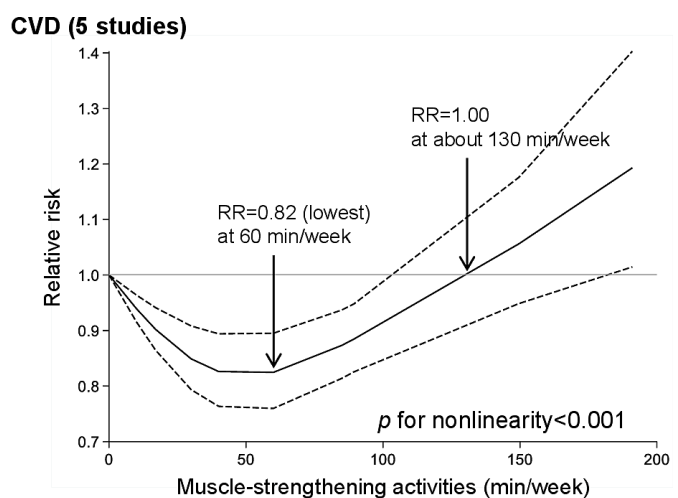

Diabetes (3 studies)

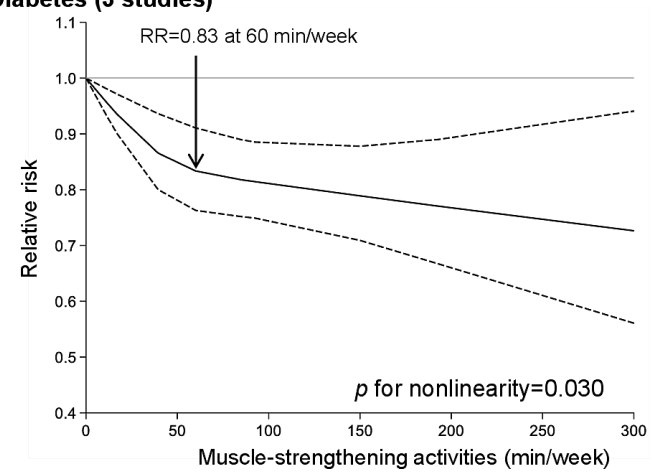

Figure 4 Non-linear dose-response meta-analysis of the associations between muscle-strengthening activities and all-cause mortality, cardiovascular disease (CVD), total cancer and diabetes. Muscle-strengthening activities were modelled with restricted cubic splines in a randomeffects dose-response model. The black line indicates the spline model and dashed lines represent $95 \%$ confidence intervals. RR, relative risk.
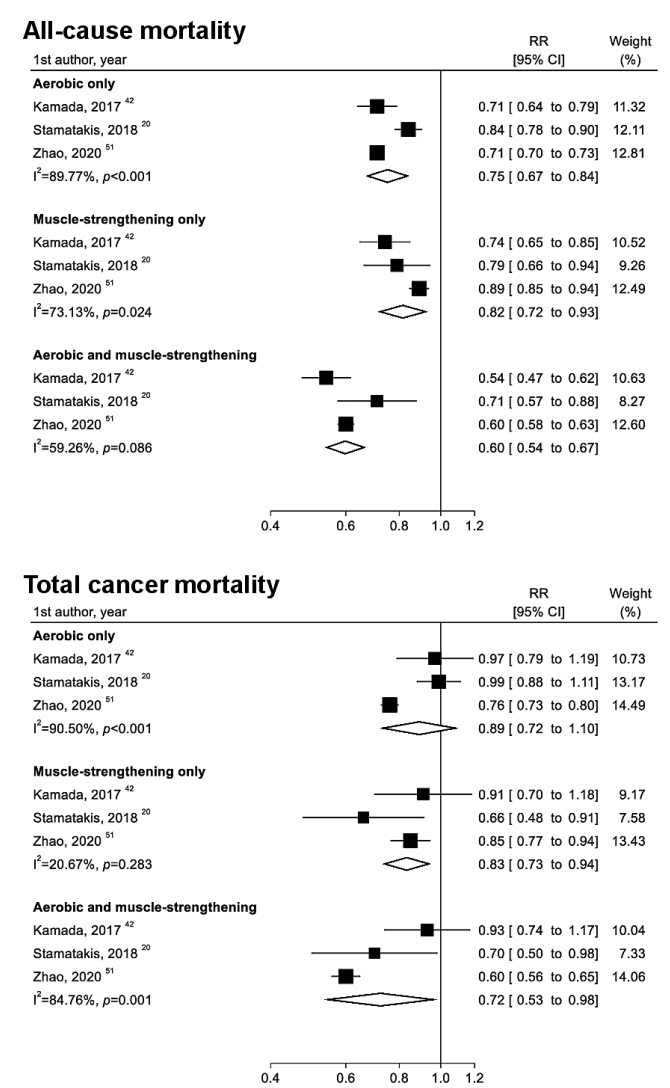
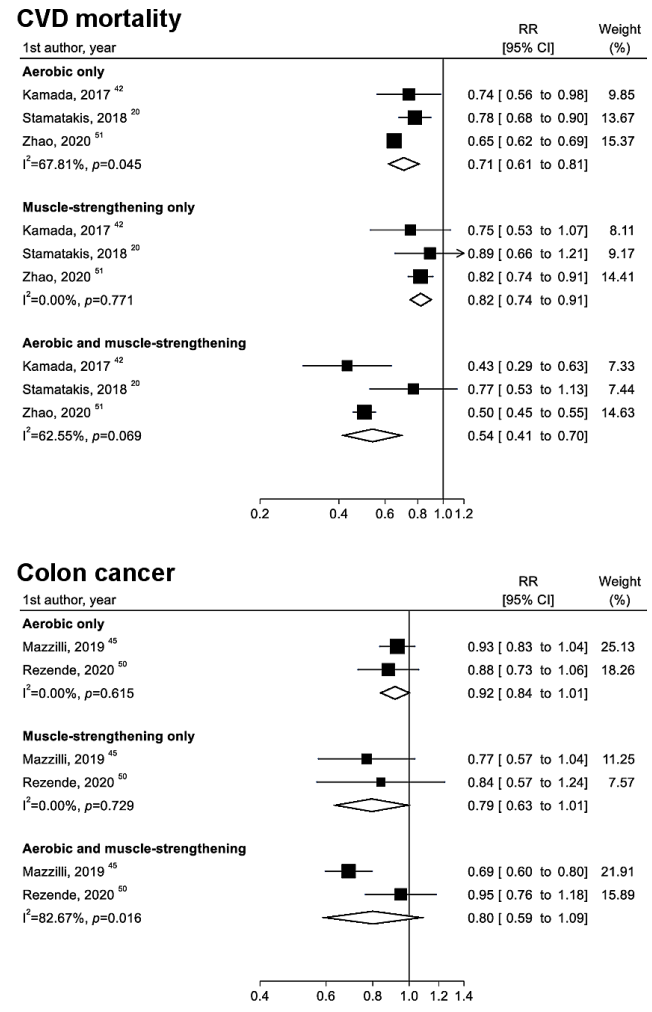

Figure 5 Meta-analysis of the joint associations of muscle-strengthening and aerobic activities with all-cause mortality, cardiovascular disease (CVD) mortality, total cancer mortality and colon cancer incidence. The definitions of groups for muscle-strengthening and aerobic activities were based on the categories described in online supplemental table 6 . RR, relative risk. 
heterogeneity $\left(\mathrm{I}^{2}=75.8 \% ; \mathrm{p}<0.001\right)$ (figure 2$)$. The exclusion of any individual study did not substantially change this result, and the high heterogeneity was not explained by the quality score or exposure assessment (online supplemental figures 1-3). When the analysis was limited to total cancer mortality (ie, excluding the study by Rezende et $a l^{50}$ ), a similar result was obtained (online supplemental figure 1).

Four studies were eligible for the dose-response analysis of muscle-strengthening exercise per $10 \mathrm{~min} /$ week increase, with a total of 212323 participants and 13033 cases. Although there was no linear association (figure 3), a non-linear association was observed (figure 4). The lowest RR (RR 0.91; 95\% CI 0.85 to 0.97) was observed at $30 \mathrm{~min} /$ week of muscle-strengthening activities and the RR estimate for up to approximately $130 \mathrm{~min} /$ week was $<1.00$.

Three studies examined the joint benefit of musclestrengthening and aerobic activities for total cancer mortality, with a total of 585930 participants and 17212 cases. Combined muscle-strengthening and aerobic activities were associated with a $28 \%$ lower risk of total cancer mortality (RR $0.72 ; 95 \%$ CI 0.53 to $0.98 ; \mathrm{I}^{2}=84.8 \%$ ) (figure 5 ).

The overall quality of the evidence on total cancer was rated as 'very low'.

\section{Diabetes}

Five studies with 9548 cases of diabetes among 202486 participants were included in the two-group analysis. Musclestrengthening activities were associated with a $17 \%$ lower incidence of diabetes (RR $0.83 ; 0.77$ to $0.89 ; \mathrm{p}<0.001$ ), with a low to moderate level of heterogeneity $\left(\mathrm{I}^{2}=35.8 \% ; \mathrm{p}=0.18\right)$ (figure 2$)$. The heterogeneity was substantially reduced $\left(\mathrm{I}^{2}=9.5 \%\right)$ when the study by Mielke et $a l^{17}$ with low quality $(\mathrm{NOS}=4)$ was excluded (online supplemental figure 1). An inverse association was obtained when the analysis was limited to studies focused on women (two studies) (online supplemental figure 5).

Three studies were eligible for the dose-response analysis of muscle-strengthening activities per $10 \mathrm{~min} /$ week increase, with a total of 167072 participants and 7511 cases. Each 10 min/week increase in muscle-strengthening activities was inversely associated with the risk of diabetes, with moderate evidence of heterogeneity (RR $0.98 ; 95 \%$ CI 0.97 to $0.99 ; \mathrm{p}=0.003 ; \mathrm{I}^{2}=58.7 \%$; $\mathrm{p}=0.09$ ) (figure 3). Moreover, an L-shaped relationship was found, and the risk markedly decreased until up to $60 \mathrm{~min} /$ week of muscle-strengthening activities (figure 4).

The overall quality of the evidence on diabetes was rated as 'low'.

\section{Site-specific cancers}

Two studies were included in the two-group and dose-response analyses for the incidence of site-specific cancers (colon, kidney, bladder, lung and pancreatic cancers). ${ }^{45} 50$ The total number of cases/participants was 2415/248 909 for colon cancer, 1063/248 909 for kidney cancer, 2341/248 909 for bladder cancer, 4075/248 909 for lung cancer and 1028/248 909 for pancreatic cancer. Muscle-strengthening activities were associated with a $10 \%$ lower incidence of lung cancer (RR $0.90 ; 95 \%$ CI 0.83 to $0.98 ; \mathrm{p}=0.01 ; \mathrm{I}^{2}=0.0 \% ; \mathrm{p}=0.69$ ) (online supplemental figure 6). A linear association was obtained for lung cancer (RR 0.99; $95 \%$ CI 0.98 to $1.00 ; \mathrm{p}=0.045 ; \mathrm{I}^{2}=0.0 \% ; \mathrm{p}=0.81$ ) (online supplemental figure 7). For other site-specific cancers, no association was confirmed in the two-group, dose-response and joint analyses (figure 5 and online supplemental figures 6 and 7).
Sensitivity analysis and any subgroup analysis were not performed because of the small number of included studies.

The overall quality of the evidence on the incidence of each site-specific cancer was rated as 'very low'.

\section{Publication bias}

For all outcomes included in the meta-analysis, the test for funnel plot asymmetry was not performed because of the small number of included studies $(\mathrm{n} \leq 7)$.

\section{DISCUSSION}

This systematic review and meta-analysis of cohort studies found that muscle-strengthening activities were inversely associated with the risk of CVD, total cancer, diabetes, lung cancer and allcause mortality independent of aerobic activities among adults aged $\geq 18$ years without severe health conditions. Moreover, J-shaped associations were found between muscle-strengthening activities and all-cause mortality, CVD and total cancer, with the maximum risk reduction (approximately 10-20\%) at approximately 30-60 $\mathrm{min} /$ week of muscle-strengthening activities. We also observed an L-shaped association between muscle-strengthening activities and diabetes, showing a large risk reduction before $60 \mathrm{~min} /$ week. Finally, combined musclestrengthening and aerobic activities (vs none) were associated with a lower risk of all-cause, CVD and total cancer mortality.

Saeidifard et al reported that engaging in muscle-strengthening activities was associated with a lower risk of all-cause mortality, although there was no clear association with CVD mortality and total cancer mortality. ${ }^{8}$ Moreover, another meta-analysis showed no clear association with total cancer mortality. ${ }^{9}$ Our systematic review updated the literature and expanded on previous studies, ${ }^{8} 9$ showing that muscle-strengthening activities were inversely associated with the risk of CVD, total cancer and allcause mortality. We obtained similar results when the analysis was limited to CVD and total cancer mortality. In addition, muscle-strengthening activities were associated with a lower incidence of lung cancer in our review, although Nascimento et al showed an inverse association for kidney cancer, but not lung cancer, even when the same studies were included. ${ }^{9}$ The reason for this discrepancy may be derived from the extracted effect estimates. Nascimento et al extracted the effect estimate from the highest category of muscle-strengthening activities whereas we used pooled effect estimates when the included studies had two or more exposed groups.

Joint analysis between muscle-strengthening and aerobic activities showed that a greater benefit for all-cause, CVD and total cancer mortality was obtained when these two types of activities were combined. These results confirm the findings of previous meta-analyses. ${ }^{8} 9$ Therefore, beyond aerobic activities, muscle-strengthening activities may provide additional benefits for preventing mortality.

One of the strengths of this study was the quantification of the dose-response association between muscle-strengthening activities and health outcomes. Several previous cohort studies have reported a non-linear association between muscle-strengthening activities and health outcomes. ${ }^{42-4448}$ For example, Kamada et al showed a quadratic association between strength training and allcause and CVD mortality, and the lowest risk of all-cause mortality was observed at $82 \mathrm{~min} /$ week of strength training. ${ }^{42}$ Furthermore, the abovementioned previous meta-analysis reported that performing resistance training 1-2 times/week was associated with a lower all-cause mortality, but increasing the volume to $>2$ times/week was not. ${ }^{8}$ This result supports a potential non-linear 
association between muscle-strengthening activities and all-cause mortality. In our systematic review, J-shaped associations with the maximum risk reduction (10-20\%) at approximately 30-60 $\mathrm{min} /$ week of muscle-strengthening activities were observed for all-cause mortality, CVD and total cancer. These results suggest that optimal doses of muscle-strengthening activities for the prevention of all-cause death, CVD and total cancer may exist.

In addition, our study is the first to systematically evaluate the longitudinal association between muscle-strengthening activities and the risk of diabetes. Although the potential of muscle-strengthening activities to reduce the risk of diabetes is supported by several biological mechanisms, ${ }^{64} 65$ many of the previous studies on this topic were limited to short-term randomised controlled trials examining surrogates of diabetes. ${ }^{66}$ Our findings showed that muscle-strengthening activities were associated with a $17 \%$ lower incidence of diabetes, with the risk of diabetes sharply decreasing until up to $60 \mathrm{~min} / \mathrm{week}$ of muscle-strengthening activities followed by a gradual decrease. Because muscle-strengthening activities increase or preserve skeletal muscle mass, which has been identified as the major tissue in glucose metabolism, a clear dose-response association can be established.

Our systematic review has some limitations. The first and most important limitation is that the meta-analysis included only a small number of studies. The limited number of studies precluded some examinations. For example, it did not allow us to conduct some subgroup analyses to explain the heterogeneity in our findings and, even when performed, few studies were included. Moreover, we could not test for publication bias. Therefore, the pooled estimates in this study might have been overestimated because of potential publication bias. Second, the included studies evaluated muscle-strengthening activities using a self-reported questionnaire or the interview method. Although measures of muscle-strengthening activities have been reported to have higher reliability than those of aerobic activities, ${ }^{67}$ this may have contributed to the heterogeneity in our results. Indeed, the heterogeneities in this review were partially explained by differences in exposure assessment, although only a few studies were included. Third, because most of the included studies were conducted in the USA, the generalisability of our findings is limited. Fourth, observational studies were included in the meta-analysis and were thus potentially influenced by residual, unknown and unmeasured confounding factors. Finally, only two databases were searched, and therefore some relevant studies may have been missed.

Several physical activity guidelines recommend that adults perform muscle-strengthening activities at least twice a week. ${ }^{1-5}$ Although the recommendation is primarily based on the benefit for musculoskeletal health, ${ }^{11-13}$ these guidelines are partly supported by our results in terms of preventing premature death and NCDs. However, the influence of a higher volume of muscle-strengthening activities on health benefits is unclear. Our findings showed that the maximum risk reduction for all-cause mortality, CVD and total cancer was obtained at approximately 30-60 min/week of muscle-strengthening activities, and the RR was low for up to approximately 130-140 min/week. Given this result, the current recommendation of at least 2 days/week could be reasonable, although a higher volume may require caution. However, our findings should be interpreted with caution because the number of included studies was small and we could not directly examine the frequency of muscle-strengthening activities. Large-scale studies are needed to examine the health benefits of high-volume muscle-strengthening activities. Moreover, attention should also be paid to evidence that most programmes
What is already known?

Physical activity guidelines recommend regular musclestrengthening activities for adults, and this recommendation is primarily based on the benefits for musculoskeletal health.

- Previous meta-analyses have shown that musclestrengthening activities are associated with a decreased risk of all-cause mortality and kidney cancer, although the doseresponse association is unknown.

- Further studies are needed to update the literature and expand on previous studies that did not provide evidence on the optimal dose of muscle-strengthening activities.

What are the new findings?

- Muscle-strengthening activities were associated with a $10-17 \%$ lower risk of CVD, total cancer, diabetes, lung cancer and all-cause mortality independent of aerobic activities among adults.

- The maximum risk reduction for all-cause mortality, CVD and total cancer was obtained at approximately 30-60 min/week of muscle-strengthening activities, and the risk of diabetes sharply decreased until $60 \mathrm{~min} /$ week of muscle-strengthening activities, followed by a gradual decrease.

providing benefits for musculoskeletal health in elderly people are performed $\geq 2$ days/week. ${ }^{12}$ The longitudinal influence of muscle-strengthening activities on mortality and NCDs should be further investigated with a focus on the elderly population in future studies.

\section{CONCLUSION}

Engaging in muscle-strengthening activities was associated with a lower risk of all-cause mortality and major NCDs such as CVD, total cancer, diabetes and lung cancer. However, the influence of a higher volume of muscle-strengthening activities on allcause mortality, CVD and total cancer is unclear, considering the observed J-shaped associations. In addition, the combination of muscle-strengthening and aerobic activities may provide a greater benefit for reducing all-cause, CVD and total cancer mortality. Given that the available data are limited, further studies-such as studies focusing on a more diverse population-are needed to increase the certainty of the evidence.

Acknowledgements The authors acknowledge E Stamatakis (University of Sydney), A Grøntved (University of Southern Denmark) and G I Mielke (The University of Queensland) for providing additional data and information pertinent to their original reports. We thank R Nagatomi (Tohoku University) for supporting this literature review. We also thank the International Medical Information CenterCentre and Inforesta Co Ltd for supporting the design of the search formula for the literature review. Furthermore, we thank Editage (www.editage.jp) for English language editing.

Contributors HM and RK contributed equally to this work. HM, RK, TH and SSS have full access to all the data in this review and take full responsibility as guarantors for the integrity of the data and the accuracy of the data analysis. HM and RK conceived and designed the study. HM, RK and TH contributed to study selection, data extraction and data analyses. RK conducted the meta-analysis. HM drafted the manuscript. All authors contributed to data interpretation and critically revised the manuscript. The corresponding author attests that all listed authors meet authorship criteria and that no others meeting the criteria have been omitted.

Funding This work was supported by Ministry of Health, Labour and Welfare (MHLW) Programme Grant Number JPMH20FA1006.

Competing interests None declared. 
Patient consent for publication Not applicable.

Ethics approval This study does not involve human participants.

Provenance and peer review Not commissioned; externally peer reviewed.

Supplemental material This content has been supplied by the author(s). It has not been vetted by BMJ Publishing Group Limited (BMJ) and may not have been peer-reviewed. Any opinions or recommendations discussed are solely those of the author(s) and are not endorsed by BMJ. BMJ disclaims all liability and responsibility arising from any reliance placed on the content. Where the content includes any translated material, BMJ does not warrant the accuracy and reliability of the translations (including but not limited to local regulations, clinical guidelines, terminology, drug names and drug dosages), and is not responsible for any error and/or omissions arising from translation and adaptation or otherwise.

Open access This is an open access article distributed in accordance with the Creative Commons Attribution 4.0 Unported (CC BY 4.0) license, which permits others to copy, redistribute, remix, transform and build upon this work for any purpose, provided the original work is properly cited, a link to the licence is given, and indication of whether changes were made. See: https://creativecommons.org/ licenses/by/4.0/

\section{ORCID iDs}

Haruki Momma http://orcid.org/0000-0003-1134-0898

Ryoko Kawakami http://orcid.org/0000-0001-8211-1553

Takanori Honda http://orcid.org/0000-0002-1011-9879

Susumu S Sawada http://orcid.org/0000-0002-7272-0790

\section{REFERENCES}

1 UK Department of Health and Social Care. Physical activity guidelines in the UK: review and recommendations, 2011. Available: https://assets.publishing.service.gov. uk/government/uploads/system/uploads/attachment_data/file/833148/dh_128255. pdf [Accessed 13 Sep 2021].

2 Australia Department of Health. Make your move - sit less, be active for life - adults (18 to 64 years), 2014. Available: https://www.health.gov.au/sites/default/files/ documents/2021/03/make-your-move-sit-less-be-active-for-life-adults-18-to-64-years. pdf [Accessed 13 Sep 2021].

3 US Department of Health and Human Services. Physical activity guidelines for Americans. 2nd ed. US Department of Health and Human Services, 2018. https:// health.gov/sites/default/files/2019-09/Physical_Activity_Guidelines_2nd_edition. pdf

4 Bull FC, Al-Ansari SS, Biddle S, et al. World Health Organization 2020 guidelines on physical activity and sedentary behaviour. Br J Sports Med 2020;54:1451-62.

5 Ross R, Chaput J-P, Giangregorio LM, et al. Canadian 24-hour movement guidelines for adults aged 18-64 years and adults aged 65 years or older: an integration of physical activity, sedentary behaviour, and sleep. Appl Physiol Nutr Metab 2020;45:557-102

6 García-Hermoso A, Cavero-Redondo I, Ramírez-Vélez R, et al. Muscular strength as a predictor of all-cause mortality in an apparently healthy population: a systematic review and meta-analysis of data from approximately 2 million men and women. Arch Phys Med Rehabil 2018;99:2100-13.

7 Wu Y, Wang W, Liu T, et al. Association of grip strength with risk of all-cause mortality, cardiovascular diseases, and cancer in community-dwelling populations: a metaanalysis of prospective cohort studies. J Am Med Dir Assoc 2017;18:551.e17-551. e35.

8 Saeidifard F, Medina-Inojosa JR, West CP, et al. The association of resistance training with mortality: a systematic review and meta-analysis. Eur J Prev Cardiol 2019;26:1647-65

9 Nascimento W, Ferrari G, Martins CB, et al. Muscle-strengthening activities and cancer incidence and mortality: a systematic review and meta-analysis of observational studies. Int J Behav Nutr Phys Act 2021;18:69.

10 Ministry of Health, Labour and Welfare of Japan. Physical activity reference for health promotion 2013. Ministry of health, labour and welfare of Japan, 2013. Available: http://www.mhlw.go.jp/stf/houdou/2r9852000002xple.html [Accessed 13 Sep 2021]

11 Physical Activity Guidelines Advisory Committee. Physical Activity Guidelines Advisory Committee report, 2008. U.S. Department of Health and Human Services, 2008. Available: https://health.gov/sites/default/files/2019-10/CommitteeReport_7.pdf [Accessed 13 Sep 2021].

122018 Physical Activity Guidelines Advisory Committee. 2018 Physical Activity Guidelines Advisory Committee scientific report. U.S. Department of Health and Human Services, 2018. Available: https://health.gov/sites/default/files/2019-09/PAG Advisory_Committee_Report.pdf [Accessed 13 Sep 2021].

13 El-Kotob R, Ponzano M, Chaput J-P, et al. Resistance training and health in adults: an overview of systematic reviews. Appl Physiol Nutr Metab 2020;45:S165-79.

14 Giovannucci EL, Rezende LFM, Lee DH. Muscle-strengthening activities and risk of cardiovascular disease, type 2 diabetes, cancer and mortality: a review of prospective cohort studies. J Intern Med 2021;290:789-805.
15 Stroup DF, Berlin JA, Morton SC, et al. Meta-analysis of observational studies in epidemiology: a proposal for reporting. Meta-analysis of Observational Studies in Epidemiology (MOOSE) group. JAMA 2000;283:2008-12.

16 Page MJ, McKenzie JE, Bossuyt PM, et al. The PRISMA 2020 statement: an updated guideline for reporting systematic reviews. BMJ 2021;372:n71.

17 Mielke Gl, Bailey TG, Burton NW, et al. Participation in sports/recreational activities and incidence of hypertension, diabetes, and obesity in adults. Scand J Med Sci Sports 2020;30:2390-8.

18 Sheehan CM, Li L. Associations of exercise types with all-cause mortality among U.S adults. Med Sci Sports Exerc 2020;52:2554-62.

19 Grøntved A, Rimm EB, Willett WC, et al. A prospective study of weight training and risk of type 2 diabetes mellitus in men. Arch Intern Med 2012;172:1306-12.

20 Stamatakis E, Lee I-M, Bennie J, et al. Does strength-promoting exercise confer unique health benefits? A pooled analysis of data on 11 population cohorts with all-cause, cancer, and cardiovascular mortality endpoints. Am J Epidemiol 2018:187:1102-12.

21 Hsu B, Merom D, Blyth FM, et al. Total physical activity, exercise intensity, and walking speed as predictors of all-cause and cause-specific mortality over 7years in older men: the Concord Health and Aging in Men Project. J Am Med Dir Assoc 2018;19:216-22.

22 Wells GA, Shea B, O'Connell D. The Newcastle-Ottawa scale (NOS) for assessing the quality of nonrandomised studies in meta-analyses, 2009. Available: http://www.ohri. ca/programs/clinical_epidemiology/oxford.asp [Accessed 13 Sep 2021].

23 DerSimonian R, Laird N. Meta-analysis in clinical trials. Control Clin Trials 1986;7:177-88.

24 Higgins JPT, Thomas J, Chandler J. Cochrane Handbook for Systematic Reviews of Interventions Version 6.1 (updated September 2020), 2020. Available: www.training. cochrane.org/handbook [Accessed 13 Sep 2021].

25 Rücker G, Cates CJ, Schwarzer G. Methods for including information from multi-arm trials in pairwise meta-analysis. Res Synth Methods 2017:8:392-403.

26 Greenland S, Longnecker MP. Methods for trend estimation from summarized doseresponse data, with applications to meta-analysis. Am J Epidemiol 1992;135:1301-9.

27 Orsini N, Bellocco R, Greenland S. Generalized least squares for trend estimation of summarized dose-response data. Stata J 2006;6:40-57.

28 Aune D, Greenwood DC, Chan DSM, et al. Body mass index, abdominal fatness and pancreatic cancer risk: a systematic review and non-linear dose-response metaanalysis of prospective studies. Ann Oncol 2012:23:843-52.

29 Orsini N, Li R, Wolk A, et al. Meta-analysis for linear and nonlinear dose-response relations: examples, an evaluation of approximations, and software. Am J Epidemiol 2012;175:66-73.

30 Higgins JPT, Thompson SG, Deeks JJ, et al. Measuring inconsistency in meta-analyses. BMJ 2003;327:557-60.

31 Egger M, Davey Smith G, Schneider M, et al. Bias in meta-analysis detected by a simple, graphical test. BMJ 1997;315:629-34.

32 Sterne JAC, Sutton AJ, loannidis JPA, et al. Recommendations for examining and interpreting funnel plot asymmetry in meta-analyses of randomised controlled trials. BMJ 2011;343:d4002.

33 Guyatt G, Oxman AD, Akl EA, et al. GRADE quidelines: 1. Introduction-GRADE evidence profiles and summary of findings tables. J Clin Epidemiol 2011;64:383-94.

34 Guyatt GH, Oxman AD, Kunz R, et al. GRADE guidelines: 7. Rating the quality of evidence--inconsistency. J Clin Epidemiol 2011;64:1294-302.

35 Guyatt GH, Oxman AD, Kunz R, et al. GRADE guidelines: 8. Rating the quality of evidence--indirectness. J Clin Epidemiol 2011;64:1303-10.

36 Guyatt GH, Oxman AD, Kunz R, et al. GRADE guidelines 6 . Rating the quality of evidence--imprecision. J Clin Epidemiol 2011;64:1283-93.

37 Guyatt GH, Oxman AD, Montori V, et al. GRADE guidelines: 5. Rating the quality of evidence--publication bias. J Clin Epidemiol 2011:64:1277-82.

38 Guyatt GH, Oxman AD, Sultan S, et al. GRADE guidelines: 9. Rating up the quality of evidence. J Clin Epidemiol 2011:64:1311-6.

39 Guyatt GH, Oxman AD, Santesso N, et al. GRADE guidelines: 12. Preparing summary of findings tables-binary outcomes. J Clin Epidemio/ 2013:66:158-72.

40 Grøntved A, Pan A, Mekary RA, et al. Muscle-strengthening and conditioning activities and risk of type 2 diabetes: a prospective study in two cohorts of US women. PLOS Med 2014;11:e1001587.

41 Kuwahara K, Honda T, Nakagawa T, et al. Strength training and risk of type 2 diabetes in a Japanese working population: a cohort study. J Diabetes Investig 2015;6:655-61.

42 Kamada M, Shiroma EJ, Buring JE, et al. Strength training and all-cause, cardiovascular disease, and cancer mortality in older women: a cohort study. J Am Heart Assoc 2017;6:e007677.

43 Shiroma EJ, Cook NR, Manson JE, et al. Strength training and the risk of type 2 diabetes and cardiovascular disease. Med Sci Sports Exerc 2017;49:40-6.

44 Liu Y, Lee D-C, Li Y, et al. Associations of resistance exercise with cardiovascular disease morbidity and mortality. Med Sci Sports Exerc 2019;51:499-508.

45 Mazzilli KM, Matthews CE, Salerno EA, et al. Weight training and risk of 10 common types of cancer. Med Sci Sports Exerc 2019;51:1845-51.

46 Porter AK, Schilsky S, Evenson KR, et al. The association of sport and exercise activities with cardiovascular disease risk: the Atherosclerosis Risk in Communities (ARIC) study. J Phys Act Health 2019;16:698-705.

47 Siahpush M, Farazi PA, Wang $\mathrm{H}$, et al. Muscle-strengthening physical activity is associated with cancer mortality: results from the 1998-2011 National Health 
Interview Surveys, National Death Index record linkage. Cancer Causes Control 2019;30:663-70.

48 Patel AV, Hodge JM, Rees-Punia E, et al. Relationship between muscle-strengthening activity and cause-specific mortality in a large US cohort. Prev Chronic Dis 2020;17:E78.

49 Porter AK, Cuthbertson CC, Evenson KR. Participation in specific leisure-time activities and mortality risk among U.S. adults. Ann Epidemiol 2020;50:27-34.

50 Rezende LFM, Lee DH, Keum N, et al. Resistance training and total and sitespecific cancer risk: a prospective cohort study of 33,787 US men. Br J Cancer 2020;123:666-72.

51 Zhao M, Veeranki SP, Magnussen CG, et al. Recommended physical activity and all cause and cause specific mortality in US adults: prospective cohort study. BMJ 2020;370:m2031.

52 Tanasescu M, Leitzmann MF, Rimm EB, et al. Exercise type and intensity in relation to coronary heart disease in men. JAMA 2002;288:1994-2000.

53 Schoenborn CA, Stommel M. Adherence to the 2008 adult physical activity guidelines and mortality risk. Am J Prev Med 2011;40:514-21.

54 Zhao G, Li C, Ford ES, et al. Leisure-time aerobic physical activity, musclestrengthening activity and mortality risks among US adults: the NHANES linked mortality study. Br J Sports Med 2014;48:244-9.

55 Loprinzi PD, Davis RE. Effects of individual, combined, and isolated physical activity behaviors on all-cause mortality and CVD-specific mortality: prospective cohort study among U.S. adults. Physiol Behav 2015;151:355-9.

56 Dankel SJ, Loenneke JP, Loprinzi PD. Dose-dependent association between musclestrengthening activities and all-cause mortality: prospective cohort study among a national sample of adults in the USA. Arch Cardiovasc Dis 2016;109:626-33.

57 Dankel SJ, Loenneke JP, Loprinzi PD. Determining the importance of meeting musclestrengthening activity guidelines: is the behavior or the outcome of the behavior (strength) a more important determinant of all-cause mortality? Mayo Clin Proc 2016;91:166-74.

58 Evenson KR, Wen F, Herring AH. Associations of accelerometry-assessed and selfreported physical activity and sedentary behavior with all-cause and cardiovascular mortality among US adults. Am J Epidemiol 2016;184:621-32.

59 Kraschnewski JL, Sciamanna CN, Poger JM, et al. Is strength training associated with mortality benefits? A 15 year cohort study of US older adults. Prev Med 2016;87:121-7

60 Bakker EA, Lee D-C, Sui X, et al. Association of resistance exercise, independent of and combined with aerobic exercise, with the incidence of metabolic syndrome. Mayo Clin Proc 2017;92:1214-22.

61 Bakker EA, Lee D-C, Sui $X$, et al. Association of resistance exercise with the incidence of hypercholesterolemia in men. Mayo Clin Proc 2018;93:419-28.

62 Buras AL, Hathaway CA, Wang T, et al. The association of resistance training with risk of ovarian cancer. Cancer Med 2021;10:2489-95.

63 Nie J, Haberstroh M, Acosta T, et al. Independent and joint associations between leisure time physical activity and strength activities with mortality outcomes in older adults at least 65 years of age: a prospective cohort study. J Gerontol A Bio/ Sci Med Sci 2021;76:2122-31.

64 Pesta DH, Goncalves RLS, Madiraju AK, et al. Resistance training to improve type 2 diabetes: working toward a prescription for the future. Nutr Metab 2017;14:24

65 Widmann M, Nieß AM, Munz B. Physical exercise and epigenetic modifications in skeletal muscle. Sports Med 2019;49:509-23.

66 Aguiar EJ, Morgan PJ, Collins CE, et al. Efficacy of interventions that include diet, aerobic and resistance training components for type 2 diabetes prevention: a systematic review with meta-analysis. Int J Behav Nutr Phys Act 2014;11:2.

67 Yore MM, Ham SA, Ainsworth BE, et al. Reliability and validity of the instrument used in BRFSS to assess physical activity. Med Sci Sports Exerc 2007:39:1267-74. 